Illinois State University

ISU ReD: Research and eData

Faculty publications - Physics

Physics

$2-2010$

\title{
Bosonic analog of the Klein paradox
}

RE. Wagner

Illinois State University

M R. Ware

Illinois State University

QSu

Illinois St

Rainer Grobe

Illinois State University

Follow this and additional works at: https://ir.library.illinoisstate.edu/fpphys

Part of the Atomic, Molecular and Optical Physics Commons

\section{Recommended Citation}

Wagner, R E.; Ware, M R.; Su, Qi and Grobe, Rainer, "Bosonic analog of the Klein paradox" (2010). Faculty publications - Physics. 22. https://ir.library.illinoisstate.edu/fpphys/22

This Article is brought to you for free and open access by the Physics at ISU ReD: Research and eData. It has been accepted for inclusion in Faculty publications - Physics by an authorized administrator of ISU ReD: Research and eData. For more information, please contact ISUReD@ilstu.edu. 


\title{
Bosonic analog of the Klein paradox
}

\author{
R. E. Wagner, M. R. Ware, Q. Su, and R. Grobe \\ Intense Laser Physics Theory Unit and Department of Physics, Illinois State University, Normal, Illinois 61790-4560, USA
}

(Received 18 December 2009; published 17 February 2010)

\begin{abstract}
The standard Klein paradox describes how an incoming electron scatters off a supercritical electrostatic barrier that is so strong that it can generate electron-positron pairs. This fermionic system has been widely discussed in textbooks to illustrate some of the discrepancies between quantum mechanical and quantum field theoretical descriptions for the pair creation process. We compare the fermionic dynamics with that of the corresponding bosonic system. We point out that the direct counterpart of the Pauli exclusion principle (the central mechanism to resolve the fermionic Klein paradox) is stimulated emission, which leads to the resolution of the analogous bosonic paradox.
\end{abstract}

DOI: 10.1103/PhysRevA.81.024101

PACS number(s): 12.20.Ds, 03.65.-w

In 1927 the Swedish scientist Oskar Klein [1] analyzed the quantum-mechanical solution of the Dirac equation for an incoming electron that scatters off a one-dimensional electrostatic barrier of height $V$. He observed a nonvanishing transmission if this height exceeds twice the rest mass energy of the electron ( $V>2 \mathrm{~m} c^{2}$ ), even for incoming energies $E$ that are far below the potential height, $E<V$. This classically forbidden nonvanishing transmission has been dubbed the "Klein paradox." Two types of approaches have been used to investigate this paradox [2-12]. The first group analyzed the dynamics in purely quantum-mechanical terms, which cannot predict any of the pair creation that is unavoidably associated with the supercritical barrier by itself. The second approach used quantum field theory, which correctly describes the pair-creation process, but the effect of the incoming electron on the pair-creation process was not included. A complete description has to be quantum field theoretical and must include the incoming particle as well as the pair-creation process at the barrier.

Numerical solutions to quantum field theory were used to study the interaction of the pair-creation process at the barrier with an incoming electron with full space-time resolution $[10,11]$. In agreement with earlier predictions, it was shown that the incoming electron suppresses the pair-creation process during the time it is being (completely) reflected at the barrier. The incoming electron occupies states that are therefore no longer available for the pair-creation process. As a result of this Pauli blocking, the reflected electron gets entangled with the pair-creation process such that it cannot be described by a single-particle wave function. The suppression due to Pauli blocking is in contradiction to statements that the incoming electron can "knock-out" a positron under the barrier [4] or that the incoming electron "stimulates" [7] the pair-creation process.

While a wide number of articles have examined the Klein paradox in its historical fermionic context, an analogous analysis within the context of a bosonic system with spacetime resolution has found less attention [5,13]. There are several reasons for this. First, the threshold with regard to the electric field strength to break down the fermionic vacuum is easier to reach than that for the bosonic system. Second, an analysis would require the analysis of the Klein-Gordon equation, which is second-order in time and therefore leads to a non-Hamiltonian framework.
Due to the absence of the Pauli exclusion principle in a system that is quantized by operators satisfying commutator (and not anticommutator) relationships, one could expect the boson-antiboson pair-creation process at the barrier remains unaffected by an incoming boson. Below we will outline a theoretical framework for such an analysis and show analytically as well as numerically with space-time resolution that the incoming boson enhances the pair-creation process at the barrier, in a fashion similar to the stimulated emission of light from an excited atom [14]. Even though the Pauli exclusion principle and stimulated emission are usually discussed as completely independent concepts, we argue that they seem to be direct counterpart of each other.

While the Lorentz invariance for a fermionic system leads to the Dirac equation, the corresponding invariance for a system with zero spin leads to the Klein-Gordon equation. In order to have a formalism that is similar to that of the Dirac equation, we use the Feshbach-Villars representation [15], in which a general quantum-mechanical state is described by a two-component vector $\phi(z, t)=\left\lfloor\left\lfloor\phi_{\phi_{2}}^{\phi_{2}}\right\rfloor\right.$. In atomic units, the Klein-Gordon Hamiltonian takes the form

$$
h=\left(\sigma_{3}+i \sigma_{2}\right) p^{2} / 2+\sigma_{3} c^{2}+V(z),
$$

where $p$ is the momentum and $c(=137$ a.u. $)$ is the speed of light. To represent the barrier we use the Sauter [16] potential $V(z)=V[1+\tanh (z / W)] / 2$, where $W$ is the spatial extent of the corresponding force and $V$ the potential strength. The usual $2 \times 2$ Pauli matrices are denoted by

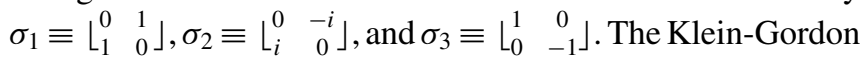
Hamiltonian [15,17] is pseudo-Hermitian $\left(h^{\dagger}=\sigma_{3} h \sigma_{3}\right)$ and consequently the total charge density $\int d z \rho(z, t)$ is conserved, where $\rho(z, t) \equiv \phi^{\dagger} \sigma_{3} \phi=\left|\phi_{1}(z, t)\right|^{2}-\left|\phi_{2}(z, t)\right|^{2}$. In contrast, under the Dirac Hamiltonian [18] the corresponding density, $\left|\phi_{1}(z, t)\right|^{2}+\left|\phi_{2}(z, t)\right|^{2}$, is always positive.

Even though pair creation needs to be described by quantum field theory, the quantum mechanical transmission coefficient $T$ plays an important role for this process. Using a uniform approach for the Dirac as well as the Klein-Gordon systems, we can define the transmission coefficient as the ratio of the transmitted to the incoming current density, $T \equiv j_{\text {trans }} / j_{\text {inc }}$, and similarly the reflection coefficient as $R \equiv-j_{\text {refl }} / j_{\text {inc }}$. The minus sign guarantees a positive coefficient $R$, as the reflected current always points in the opposite direction of 
the incoming current. As a result we always have $R+T=$ 1 , independent of the direction or charge of the incoming particle and independent of whether the system is fermionic or bosonic or whether the potential is sub- or supercritical [19]. As found by Klein, in the supercritical regime $\left(V>2 c^{2}\right)$ the transmission coefficient does not vanish for incoming energies $c^{2}<E<V-c^{2}$. In this case, $T(E)$ is positive because the time evolution is unitary and the density $\rho(z, t)$ is always positive and $0<T(E)<1$. However, the supercritical Klein-Gordon potential leads to a sign change of the charge density under the barrier relative to the incoming density, and as a result the transmission coefficient is negative and even unbounded from below, $-\infty<T(E)<0$. In this case the amount of the area under the reflected wave packet can be larger than unity $(R>1)$ such that $R+T=1$ is still valid. For a space-time-resolved comparison of the quantum mechanical Dirac and Klein-Gordon wave packet dynamics, see Ref. [20].

In the case of the Sauter potential, the transmission coefficient for the supercritical case can be obtained analytically [16], $T=\{\cosh [\pi(p-q) W]-\cosh [\pi(p+q) W]\} /$ $\left(\cosh \left\{\pi\left[(W V / c)^{2}-1\right]^{1 / 2}\right\}+\cosh [\pi(p-q) W]\right)$, where $p \equiv$ $\sqrt{ }\left(E^{2}-c^{4}\right) / c$ is the momentum of the incoming particle of energy $E$ and $q$ is the momentum the particle takes under the barrier $q \equiv \sqrt{ }\left[(E-V)^{2}-c^{4}\right] / c$. For $W \rightarrow 0$, the transmission coefficient simplifies to $T=-4 p q /(p-q)^{2}$, which can diverge if $E=V / 2$ such that $p=q$.

Despite the fact that the Dirac and Klein-Gordon transmission coefficients differ in sign and by their range of values, one can show [20-23] that for both systems, in the absence of any incoming particle, the pair-creation rate $\gamma$ (the number of created pairs per unit time) at the barrier is directly given by the universal form $\gamma=\int d E|T(E)| /(2 \pi)$, where the energy integral extends from $E=c^{2}$ to $E=V-c^{2}$. We point out, however, that our data disagree with Refs. [2,4] that derived a negative transmission coefficient for the Dirac system and also Ref. [8] that used a positive transmission coefficient for the Klein-Gordon system.

In order to examine the combination of the incoming boson with the pair-creation process, we have to use quantum field theory and calculate the time evolution of the bosonic quantum field operator $\hat{\Psi}$. The evolution of this operator $[4,22,24]$ can be obtained from either the Schrödinger-like equation, $i \partial \hat{\Psi}(t) / \partial t=h \hat{\Psi}(t)$, or the Heisenberg equation, $i \partial \hat{\Psi}(t) / \partial t=[\hat{\Psi}, \hat{H}]$. The corresponding quantum field theoretical Hamiltonian $\hat{H}$ can be obtained from its quantum mechanical limiting case $h$ via $\hat{H}=\hat{\Psi}^{\dagger} \sigma_{3} h \hat{\Psi}$.

The field $\hat{\Psi}$ can be expanded in terms of creation and annihilation operators, $\hat{\Psi}(t)=\Sigma_{p} \hat{b}_{p}|p(t)\rangle+\Sigma_{n} \hat{d}_{n}^{\dagger}|n(t)\rangle$, which commute, $\left[\hat{b}_{p}, \hat{b}_{p^{\prime}}^{\dagger}\right]=\delta_{p, p^{\prime}}$ and $\left[\hat{d}_{n}, \hat{d}_{n^{\prime}}^{\dagger}\right]=\delta_{n, n^{\prime}}$. The initial states $|p\rangle$ and $|n\rangle$ can be chosen as the quantum mechanical energy eigenstates of the force-free $(V=0)$ Hamiltonian $h$ with positive and negative energy. The time-evolved states $|p(t)\rangle=\exp [-i h t]|p\rangle$ and $|n(t)\rangle=\exp [-i h t]|n\rangle$ can be obtained numerically on a space-time lattice grid using fast Fourier transform-based split-operator techniques [25-29]. A similar but more efficient approach has been developed by Ruf et al. [30] to study the quantum mechanical version of the Klein paradox based on the Klein-Gordon equation in two spatial dimensions.
We assume that the incoming boson has a positive charge and its initial quantum field theoretical state is a superposition of single-particle states with momentum $p, \| \Phi(t=0)\rangle\rangle=$ $\left.\left.\Sigma_{p} C_{p} \hat{b}_{p}^{\dagger} \| 0\right\rangle\right\rangle$. We use the double bars to distinguish (secondquantized) quantum field states (such as the vacuum $\| 0\rangle\rangle$ ) from quantum mechanical states, such as $|p\rangle$ or $|n\rangle$. We choose Gaussian expansion coefficients $C_{p}$ such that the corresponding initial spatial density $\rho_{+}(z)=\left\langle z\left|\left\langle\left\langle\Phi\left\|\hat{\Psi}_{+}^{\dagger} \sigma_{3} \hat{\Psi}_{+}\right\| \Phi\right\rangle\right\rangle\right| z\right\rangle$ is localized to the left of the barrier. Here the subscript + denotes the positive frequency part [24] of the operator $\hat{\Psi}$, defined by the projection $\hat{\Psi}_{+}(t)=\Sigma_{p}|p\rangle\langle p| \sigma_{3} \hat{\Psi}(t)$. If we insert the solution for $\hat{\Psi}(t)$ in terms of the solutions $|p(t)\rangle$ and $|n(t)\rangle$ into these definitions, we obtain after some lengthy algebra

$$
\begin{aligned}
& \rho_{+}(z, t) \\
& =\Sigma_{p 1} \Sigma_{p 2} \Sigma_{p 3} \Sigma_{p 4} C_{p 2} C_{p 4}^{*}\left\langle p_{1}\left|\sigma_{3}\right| p_{2}(t)\right\rangle\left\langle p_{3}\left|\sigma_{3}\right| p_{4}(t)\right\rangle^{*} \\
& \quad \times\left(\left\langle z \mid p_{1}\right\rangle \sigma_{3}\left\langle z \mid p_{3}\right\rangle^{*}\right)+\Sigma_{p 1} \Sigma_{p 2} \Sigma_{n}\left\langle p_{1}\left|\sigma_{3}\right| n(t)\right\rangle \\
& \quad \times\left\langle p_{2}\left|\sigma_{3}\right| n(t)\right\rangle^{*}\left(\left\langle z \mid p_{1}\right\rangle \sigma_{3}\left\langle z \mid p_{2}\right\rangle^{*}\right) \\
& \rho_{-}(z, t) \\
& =\Sigma_{p 1} \Sigma_{n 1} \Sigma_{p 2} \Sigma_{n 2} C_{p 1} C_{p 2}^{*}\left\langle n_{1}\left|\sigma_{3}\right| p_{1}(t)\right\rangle\left\langle n_{2}\left|\sigma_{3}\right| p_{2}(t)\right\rangle^{*} \\
& \quad \times\left(\left\langle z \mid n_{1}\right\rangle \sigma_{3}\left\langle z \mid n_{2}\right\rangle^{*}\right)+\Sigma_{n 1} \Sigma_{n 2} \Sigma_{p}\left\langle n_{1}\left|\sigma_{3}\right| p(t)\right\rangle \\
& \quad \times\left\langle n_{2}\left|\sigma_{3}\right| p(t)\right\rangle^{*}\left(\left\langle z \mid n_{1}\right\rangle \sigma_{3}\left\langle z \mid n_{2}\right\rangle^{*}\right) .
\end{aligned}
$$

The expression for the antiboson $\rho_{-}(z, t)$ can be obtained similarly from the charge conjugated negative frequency part, defined as $\hat{\Psi}_{-}(t)=\Sigma_{n}|C n\rangle\langle C n| \sigma_{3} C \hat{\Psi}(t)$, with the charge conjugation operator $C=\sigma_{1} K$, where $K$ is the complex conjugation operator.

Let us now give a specific numerical example. The charge density of the incoming boson $\rho_{0}(z)$ is shown in Fig. 1 by the dotted line. It has an initial width $\Delta z_{0}=8 \times 10^{-3}$ a.u., it is localized at $z_{0}=-0.4$ a.u., and it has an incoming velocity of $v=0.92 c$ corresponding to an energy of $E=2.5 c^{2}$. The barrier is centered at $z=0$ with a strength $V=5 c^{2}$ and a spatial width of $W=0.9 / c$.

To better judge the impact of the incoming boson on the pair-creation process, we show by the dashed line in the figure the spatial charge density of the created bosons in the absence of any incoming particle. We have assumed that the barrier was turned on at time $t=0$ and graph the density at time $t=4.86 \times 10^{-3}$ a.u. As the left edge (wave front) can evolve with the maximum speed $c$, it can move at most to position $z=c t(\approx-0.66$ a.u $)$. The total number of created bosons is given by the area of the density, which amounts to 14.74. We will discuss the growth of the number of pairs as a function of time below. The corresponding density of the negatively charged antibosons (evolving to the right under the barrier and not shown in the figure) is the mirror image of the boson density.

The graph for $z>0$ represents the negative charge density $\rho_{-}(z)$ of the created antibosons in the presence of the incoming boson. The peak at $z \approx 0.2$ a.u. is due to the temporary enhancement of the pair-creation process around the time $t=z_{0} / v\left(\approx 3.2 \times 10^{-3}\right.$ a.u. $)$ when the incoming boson reflects at the barrier. The area of the peak itself is 0.42 , leading to the total number of created antibosons $N_{-}=14.74+0.42=$ 15.16. The shape of this pair-creation enhancement peak is 


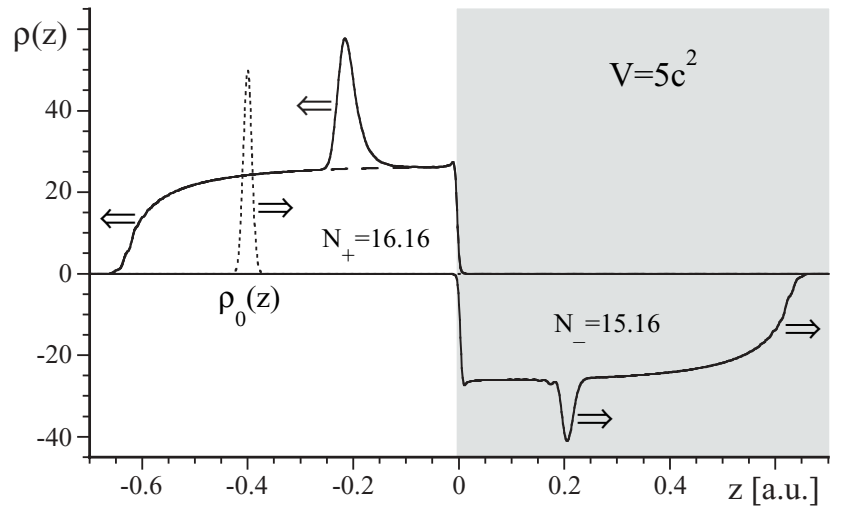

FIG. 1. The final charge distribution of the bosons $\rho_{+}(z, t)$ for $z<0$ and antibosons $\rho_{-}(z, t)$ for $0<z$ at time $t=4.86 \times 10^{-3}$ a.u. as created by a supercritical potential step $V=5 c^{2}$ in the presence of an incoming boson. The dotted line is the initial distribution of the incoming boson, $\rho_{0}(z)$. The numbers $N_{ \pm}$indicate the areas under the two densities. For comparison, the dashed line for $z<0$ shows the density created at the barrier in the absence of any incoming boson with an area of 14.74. [numerical lattice with 6000 temporal $\left(\Delta t=9.73 \times 10^{-7}\right.$ a.u. $)$ and 2048 spatial $\left(\Delta z=1.37 \times 10^{-3}\right.$ a.u. $)$ grid points, initial incoming wave packet has a spatial width $\Delta z_{0}=$ $8 \times 10^{-3}$ a.u. and energy $E=2.5 c^{2}$, the potential barrier has a width $W=6.57 \times 10^{-3}$ a.u.]

identical to the spatial profile of the transmitted quantum mechanical wave function in the absence of any pair creation. The area is therefore identical to the transmission coefficient for the incoming energy. Using the above expression derived by Sauter for the specific parameters $\left(V=5 c^{2}, W=0.9 / c\right.$, $E=V / 2)$, the numerical value of the transmission coefficient at the central energy of the incoming packet $T(E)(=-0.437)$, closely matches the negative area of the transmitted wave packet portion. As the transmission coefficient $T(E)$ has no lower bound the amount of enhancement can be arbitrarily large. For example, for a vanishing width of the barrier, $W=0$, we obtain $T(E=V / 2)=-\infty$, which suggests the possibility of, at least in principle, an infinite enhancement. If the potential force could be maintained this divergent behavior might be observable. However, it is presently not known whether to use external potentials with arbitrarily small widths $W$ in quantum field theory is still a valid approximation. We believe that there is no analogous phenomenon in traditional stimulated emission because the number of generated photons is limited by the number of excited atoms. As a photon is identical to its own antiparticle the distinction between bosons and antibosons is also absent.

The final density of the created bosons $\rho_{+}(z, t)$ has a similar peak; however, its area reflects the enhancement $(0.42)$ as well as the reflected boson (1.0) such that the total number of bosons on the left side of the barrier is $14.74+0.42+$ $1=16.16$. We note that the area of the reflected portion of the corresponding quantum mechanical calculation is $R(E=$ $V / 2)=1.437$. The spatial shape of the peak has developed an interesting asymmetric form. This steepening of the wave front is a purely relativistic effect as the packet's spreading speed at the front edge is limited by $c[31,32]$.

Let us finish this note by analyzing the temporal growth of the total number of created particle pairs. By integrating over the spatial densities obtained in Eq. (2), we can calculate the total number of bosons $N_{+}(t)=\int d z \rho_{+}(z, t)$ and antibosons $N_{-}(t)=-\int d z \rho_{-}(z, t)$. Equivalently, we can also use the time-evolved state $\| \Phi(t)\rangle\rangle$ to determine the total number of pairs. If we switch from the Schrödinger to the Heisenberg representation, the time evolution can be expressed by the field operator, leading to

$$
N_{+}(t)=\int d z\left\langle z\left|\left\langle\left\langle\Phi|| \hat{\Psi}_{+}^{\dagger}(t) \sigma_{3} \hat{\Psi}_{+}(t)|| \Phi\right\rangle\right\rangle\right| z\right\rangle .
$$

We can insert the time evolution for $\hat{\Psi}_{+}(t)=\Sigma_{p^{\prime}}\left[\Sigma_{p} \hat{b}_{p}\right.$ $\left.\left\langle p^{\prime} \mid \sigma_{3} p(t)\right\rangle+\Sigma_{n} \hat{b}_{n}^{\dagger}\left\langle p^{\prime} \mid \sigma_{3} n(t)\right\rangle\right]\left|p^{\prime}\right\rangle$ and correspondingly for $\hat{\Psi}_{+}^{\dagger}(t)$ into Eq. (3) and we obtain after a lengthy calculation

$$
N_{+}(t)=1+\Sigma_{n, p}\left|\left\langle n \mid \sigma_{3} p(t)\right\rangle\right|^{2}+\Sigma_{n}\left|\Sigma_{p} C_{p}\left\langle n \mid \sigma_{3} p(t)\right\rangle\right|^{2}
$$

and $N_{-}(t)=N_{+}(t)-1$. At each time they differ by unity reflecting the presence of the incoming boson. Note that $\int d z\left[\rho_{+}(z, t)+\rho_{-}(z, t)\right]$ represents the total charge and remains unity for the entire evolution; also the total number of pairs $N(t)$ is equal to $N_{-}(t)$. The second term in Eq. (4) is the number of particles that would have been created if there were no incoming boson and the state were initially the vacuum, $\| \Phi(0)\rangle\rangle=\| 0\rangle\rangle$; in other words, it describes the number of pairs that are created at the barrier independent of any incoming particle. In a previous work [22] it was shown that in the long-time limit $\Sigma_{n, p}\left|\left\langle n \mid \sigma_{3} p(t)\right\rangle\right|^{2}=\gamma t$, where $\gamma$ is directly related to the transmission coefficient as shown above for the Dirac as well as Klein-Gordon systems. The third term in Eq. (4) amounts to a correction of the total number of pairs due to the incoming particle. The plus sign indicates that the incoming boson enhances the pair creation at the barrier [5].

We note that the corresponding derivation for the Dirac system leads to formally identical expressions $[10,11]$ (except there is no $\sigma_{3}$ ); however, the sign in front of the third term is negative, reflecting the suppressive effect associated with

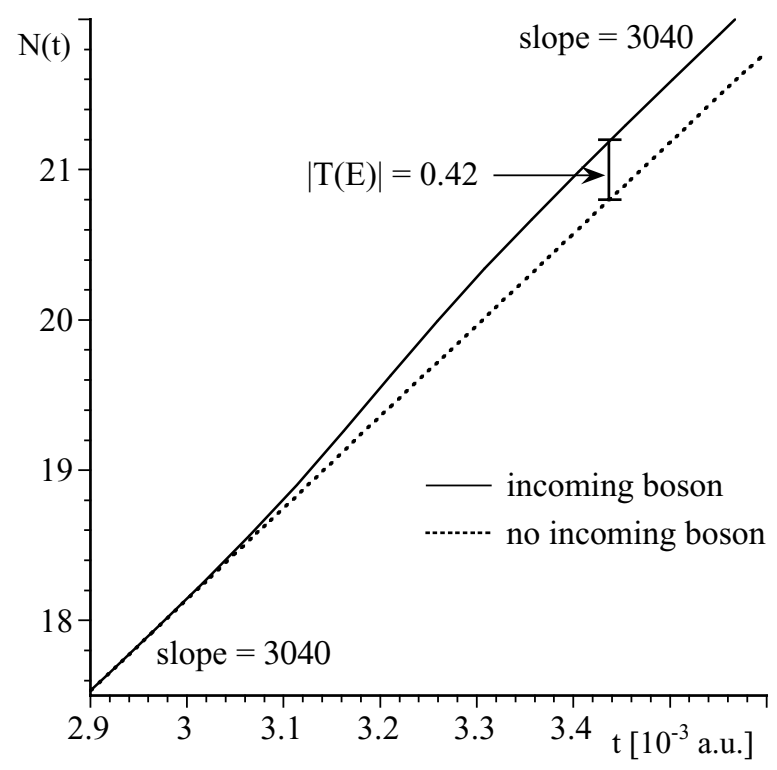

FIG. 2. The total number of created boson pairs $N(t)$ as a function of time during the time interval when the incoming boson reflects at the boundary. For comparison, the dashed line is the number in the absence of any incoming boson. (All parameters as in Fig. 1.) 
the Pauli exclusion principle. This striking similarity between both expressions shows that the (fermionic) Pauli exclusion principle and the (bosonic) stimulated emission effect are directly analogous to each other.

Except during a short time interval associated with the sudden turn-on of the potential, the number of created bosons grows linearly. $N(t)$ increases during the time interval in which the incoming boson interacts with the pair-creation process, and once the boson is completely reflected at the barrier the growth rate returns to its "unenhanced" linear growth. In Fig. 2 we show $N(t)$ as a function of time during the time interval [centered around $t \approx 3.2 \times 10^{-3}$ a.u.] when the wave packet reflects from the barrier. The duration of the interval is directly proportional to the spatial width of the incoming boson. As we discussed above, this rate can be obtained directly from the energy integral over the transmission coefficient and for our parameters we obtain $\gamma=3040.96$, which is in excellent agreement with the numerical value of 3040 found via the slope of the data. Also the effective off-set of 0.42 is close to $T(E)$.
In summary, we have shown that the bosonic Kleinparadox can be explained in terms of stimulated emission. The quantum mechanical description of the fermionic and bosonic systems in terms of the Dirac and Klein-Gordon equations are substantially different in several ways. The Dirac theory is unitary leading to positive definite densities and bounded transmission coefficients $(0<T<1)$. The Klein-Gordon theory is pseudounitary, the charge density changes its sign as it is partially transmitted and the transmission coefficient has no lower bound $(-\infty<T<0)$. The quantum field theoretical description, however, leads to a very similar formalism, except with different signs, identifying the Pauli exclusion principle and stimulated emission as direct dual mechanisms of each other.

\section{ACKNOWLEDGMENTS}

We thank Dr. T. Cheng for many helpful discussions. This work has been supported by the NSF. We also acknowledge support from the Research Corporation.
[1] O. Klein, Z. Phys. 53, 157 (1929); 41, 407 (1927).

[2] J. D. Bjorken and S. D. Drell, Relativistic Quantum Mechanics (McGraw Hill, New York, 1964), p. 42.

[3] A. Hansen and F. Randal, Phys. Scr. 23, 1036 (1981).

[4] W. Greiner, B. Müller, and J. Rafelski, Quantum Electrodynamics of Strong Fields (Springer Verlag, Berlin, 1985).

[5] C. A. Manogue, Ann. Phys. 181, 261 (1988).

[6] B. R. Holstein, Am. J. Phys. 66, 507 (1998).

[7] H. Nitta, T. Kudo, and H. Minova, Am. J. Phys. 67, 966 (1999).

[8] M. Merad, L. Chetouni, and A. Bounames, Phys. Lett. A267, 225 (2000).

[9] N. Dombey, P. Kennedy, and A. Calogeracos, Phys. Rev. Lett. 85, 1787 (2000).

[10] P. Krekora, Q. Su, and R. Grobe, Phys. Rev. Lett. 92, 040406 (2004).

[11] P. Krekora, Q. Su, and R. Grobe, Phys. Rev. A 72, 064103 (2005).

[12] P. Hejcik and T. Cheon, Europhys. Lett. 81, 50001 (2008).

[13] For early work on bosonic and fermionic pair creation rates, see E. Brezin and C. Itzykson, Phys. Rev. D 2, 1191 (1970); N. B. Narozhny and A. I. Nikishov, Sov. Phys. JETP 38, 427 (1974).

[14] For a review, see, e.g., W. P. Schleich, Quantum Optics in Phase Space (Wiley-VCH, Berlin, 2001); C. C. Gerry and P. L. Knight, Introductory Quantum Optics (Cambridge University Press, New York, 2005).

[15] H. Feshbach and F. Villars, Rev. Mod. Phys. 30, 24 (1958).

[16] F. Sauter, Z. Phys. 69, 742 (1931); 73, 547 (1931).
[17] For a review on the properties of the Klein-Gordon Hamiltonian, see, e.g., W. Greiner, Relativistic Quantum Mechanics, 3rd ed. (Springer, Berlin, 2000); A. Wachter, Relativistische Quantenmechanik (Springer, Berlin, 2005).

[18] For a review of the one-dimensional Dirac equation, see, e.g., T. Cheng, Q. Su, and R. Grobe, Contemp. Phys. [in press].

[19] For works with different conventions, see Refs. [2,4,8].

[20] T. Cheng, M. R. Ware, Q. Su, and R. Grobe, Phys. Rev. A 80, 062105 (2009).

[21] F. Hund, Z. Phys. 117, 1 (1941).

[22] T. Cheng, Q. Su, and R. Grobe, Phys. Rev. A 80, 013410 (2009).

[23] T. Cheng, C. C. Gerry, Q. Su, and R. Grobe, Europhys. Lett. 86, 13001 (2009).

[24] For a review, see, e.g., S. S. Schweber, An Introduction to Relativistic Quantum Field Theory (Harper \& Row, New York, 1962).

[25] J. A. Fleck, J. R. Morris, and M. D. Feit, Appl. Phys. 10, 129 (1976).

[26] A. D. Bandrauk and H. Shen, J. Chem. Phys. 99, 1185 (1993).

[27] U. W. Rathe, C. H. Keitel, M. Protopapas, and P. L. Knight, J. Phys. B 30, L531 (1997).

[28] J. W. Braun, Q. Su, and R. Grobe, Phys. Rev. A 59, 604 (1999).

[29] G. R. Mocken and C. H. Keitel, Comput. Phys. Commun. 178, 868 (2008).

[30] M. Ruf, H. Bauke, and C. H. Keitel, J. Comput. Phys. 228, 9092 (2009).

[31] Q. Su, B. A. Smetanko, and R. Grobe, Laser Physics 8, 93 (1998).

[32] Q. Su, B. A. Smetanko, and R. Grobe, Opt. Express 2, 277 (1998). 